\title{
Neuroprotective Effect of Plasminogen Activator Inhibitor-1 Antagonist in the Rat Model of Mild Traumatic Brain Injury
}

Pınar Kuru Bektaşoğlu ( $\sim$ pnr.kuru@gmail.com )

Marmara University Institute of Health Sciences https://orcid.org/0000-0001-9889-9955

Türkan Koyuncuoğlu

Marmara University School of Medicine

Selin Akbulut

Marmara University School of Medicine

Dilek Akakın

Marmara University School of Medicine

İrem Peker Eyüboğlu

Marmara University School of Medicine

Can Erzik

Marmara University School of Medicine

Meral Yüksel

Marmara University Vocational School of Health Services

Hızır Kurtel

Marmara University School of Medicine

\section{Research Article}

Keywords: antioxidant, antiinflammatory, neuroprotection, plasminogen activator inhibitor-1 antagonist, traumatic brain injury.

Posted Date: May 7th, 2021

DOl: https://doi.org/10.21203/rs.3.rs-479179/v1

License: (c) (i) This work is licensed under a Creative Commons Attribution 4.0 International License. Read Full License

Version of Record: A version of this preprint was published at Inflammation on August 30th, 2021. See the published version at https://doi.org/10.1007/s10753-021-01520-0. 


\section{Abstract}

Background: Plasminogen activator inhibitor-1 (PAl-1) antagonists are known for their neuroprotective effects. In this study, it was aimed to investigate the possible protective effects of PAl-1 antagonists in a rat mild traumatic brain injury (TBI) model.

Method: Sprague Dawley male rats were grouped as sham $(n=7)$, TBI $(n=9)$, TBI + PAI- 1 antagonist (5 and 10 mg/kg TM5441 and TM5484; n=6-7). Under anesthesia, TBI was induced by dropping a metal 300gram weight from a height of 1 meter on the skull. Before and 24-hour after trauma neurological examination, tail suspension, Y-maze, novel object recognition tests were performed. Twenty-four hours after $\mathrm{TBI}$, the rats were decapitated and activities of myeloperoxidase, nitric oxide release, luminol- and lucigenin-enhanced chemiluminescence were measured. Also, interleukin- $1 \mathrm{~b}$, interleukin-6, tumor necrosis factor, interleukin-10, tumor growth factor-b, caspase-3, cleaved caspase-3, and PAl levels were measured with the ELISA method in the brain tissue. Brain injury was graded histopathologically following hematoxylin-eosin staining. Western blot and immunohistochemical investigation for low-density lipoprotein receptor, matrix metalloproteinase-3 and nuclear factor-kB were also performed. Data were analyzed using GraphPad Prism 8.0 (GraphPad Software, San Diego, CA, USA) and expressed as means \pm SEM. Values of $p<0.05$ were considered to be statistically significant.

Results: Higher levels of myeloperoxidase activity in the TBI group $(p<0.05)$ were found to be suppressed in 5 and $10 \mathrm{mg} / \mathrm{kg}$ TM5441 treatment groups $(p<0.05-p<0.01)$. The tail suspension test score was increased in the TBI group ( $p<0.001)$, and decreased in all treatment groups $(p<0.05-0.001)$. The histologic damage score was increased statistically significantly in the cortex, dentate gyrus, and CA3 regions in the TBI group $(p<0.01-0.001)$, decreased in the treatment groups in the cortex and dentate gyrus $(p<0.05-0.001)$.

Conclusion: PAI antagonists, especially TM5441, has antioxidant and anti-inflammatory properties against mild TBI in the acute period. Behavioral test results were also improved after PAI antagonist treatment after mild TBI.

\section{Highlights}

After mild TBI, PAl-1 antagonists TM5441 and TM5484 was used for the first time in the literature.

PAl-1 inhibition reduced oxidative stress, inflammation and neuronal damage in a TBI model.

PAl antagonist treatment also improved corticospinal pathway functions and behavioral results.

\section{Introduction}

Traumatic brain injury (TBI) is defined as a change in brain function, or the presence of another brain pathology as a result of an external force [1]. TBI is one of the main causes of disability and death 
worldwide, especially in the young population [2-5]. At least 2-5 million new cases of TBI occur each year in European countries. 5 In the USA, it is estimated that 52,000 people die annually and 530,000 people experience disability due to TBI [6]. TBI is a process, not a static injury, and long-term symptoms in TBI survivors are known to cause structural and functional damage. It is estimated that 10 million people in the USA and Europe experience permanent disability and cognitive impairment due to TBI [7, 8]. TBI also causes neurological diseases such as epilepsy, Alzheimer's disease, Parkinson's disease, and chronic neuritis in years [9]. Patients and their caregivers can experience serious economic and moral consequences [5]. TBI can be classified as mild, moderate, or severe according to the patient's neurological signs and symptoms [5]. As an estimate, $75-90 \%$ of all TBI patients are categorized as mild TBI $[10,11]$.

The effects of $\mathrm{TBI}$ are divided into primary and secondary injuries. Primary injury is the mechanical damage that develops at the time of trauma and occurs directly in neurovascular structures and glial cells. Secondary injury begins minutes after the trauma and all available treatment modalities are directed at the secondary injury. Secondary injury is associated with pathophysiological mechanisms such as excitotoxicity, ionic imbalance due to lack of energy, inflammation, oxidative stress, and apoptosis $[12,13]$. Studies aimed at treating TBI have often focused on neuroprotective agents, and a drug that has reached clinical efficacy has not yet been found $[14,15]$.

PAl-1 is a key endogenous inhibitor of plasmin-mediated fibrinolysis and increases clot formation after injury [16]. It is a member of the serine protease inhibitor superfamily [17]. PAl-1 is found in plasma, vascular endothelium, liver, adipose tissue, neutrophils, astrocytes, and platelets [18, 21]. It provides a tight balance between clot formation and clot breakdown. Normally, clot formation begins when a tissue factor is released following the damage of the vessels and the parenchyma [22]. At the same time, tissue plasminogen activator (tPA) is also released from the damaged endothelium to prevent clot formation and vascular occlusion [23]. PAI-1, which binds to active centers of TPA and uPA, causes irreversible inhibition of these fibrinolytic molecules $[24,25]$. Because tPA is used for thrombolytic purposes in various medical conditions such as cerebral infarction and embolism, it is logical to assume that inhibition of known tPA inhibitors such as PAI-1 would be beneficial in preventing clot formation. However, PAl-1 is not just a tPA or a uPA inhibitor and has diverse biological actions, and its production increases under some pathological conditions. Various inflammatory stimuli and mediators induce PAI-1 release [26].

PAl-1 has clinical significance in pathologies accompanied by ischemia, inflammation, and vascular dysfunction [16, 27]. High PAl-1 levels after ischemic stroke is a risk factor and adversely affect survival $[28,29]$. PAl-1 deficiency has been reported to reduce brain damage in an experimental ischemic stroke model [16]. It was reported that PAl-1 is associated with increased coagulation response after TBI, and the absence or inhibition of PAI-1 reduces the lesion area after TBI [17]. In a human study, an increase in PAl-1 levels observed after head trauma and has been associated with poor prognosis [30]. Twelve hours after TBI the PAI mRNA level reaches its peak value. Demonstration of a role for PAl-1 in the pathophysiology of inflammation as well as cerebrovascular injury drove considerable attention to the development of PAI- 
1 antagonists and no studies are testing the possible anti-inflammatory actions of PAl-1 antagonists during the secondary brain injury after TBI.

In this study, the possible neuroprotective effects of PAI-1 antagonism against mild TBI were investigated with various parameters. It is thought that the administration of PAI- 1 antagonists after mild TBI will show neuroprotective activity by reducing inflammation, oxidative stress, and apoptosis, and will have a positive impact on corticospinal tract functions and learning. Since there are no comprehensive studies evaluating treatment responses in behavioral tests that deteriorate after TBI, another objective of this study is to investigate the PAl-1 antagonism on behavioral tests after mild TBI.

\section{Materials And Methods}

All experimental procedures used in this investigation were reviewed and approved by the Marmara University Animal Care and Use Committee (12.2019.mar). Animal care and all experiments were conducted in concordance with the European Communities Council Directive of November 24, 1986 $(86 / 609 / E E C)$ on the protection of animals for experimental use. The ARRIVE guidelines was applied for this study. Forty-one adult male Sprague Dawley rats weighing $250-400 \mathrm{~g}$ were used. Animals were housed in an air-conditioned room with 12-h light and dark cycles maintained at constant temperature $\left(22 \pm 2^{\circ} \mathrm{C}\right)$ and relative humidity $(65-70 \%)$. Rats were fed standard laboratory chow and had free access to water.

The rats were randomly assigned to four groups as follows:

1. Sham group $(n=7)$ : Rats underwent only a skin incision under anesthesia and received a single intragastric dose of saline $(0.9 \% \mathrm{NaCl}, 0.1 \mathrm{ml} / 100 \mathrm{~g}) 15$ minutes after surgery.

2. TBI group ( $(\mathbf{=} 9)$ : Rats underwent TBI as described below and received a single intragastric dose of saline $(0.9 \% \mathrm{NaCl}, 0.1 \mathrm{ml} / 100 \mathrm{~g}) 15$ minutes after TBI.

3. TM5441-5 mg group $(n=6)$ : Rats underwent TBI as described below and received a single intragastric dose of TM5441 (5 mg/kg in 0.5\% carboxymethylcellulose, Tohoku University Miyagi, Japan) 15 minutes after TBI [31].

4. TM5441-10 mg group ( $n=6$ ): Rats underwent TBI as described below and received a single intragastric dose of TM5441 (10 mg/kg in 0.5\% carboxymethylcellulose, Tohoku University Miyagi, Japan) 15 minutes after TBI.

5. TM5484-5 mg group $(n=6)$ : Rats underwent TBI as described below and received a single intragastric dose of TM5484 (5 mg/kg in 0.5\% carboxymethylcellulose, Tohoku University Miyagi, Japan) 15 minutes after TBI [32].

6. TM5484-10 mg group ( $n=7$ ): Rats underwent TBI as described below and received a single intragastric dose of TM5484 (10 mg/kg in 0.5\% carboxymethylcellulose, Tohoku University Miyagi, Japan) 15 minutes after TBI. 


\subsection{Anesthesia and induction of TBI}

The animals were anesthetized by an intraperitoneal injection of $0.5 \mathrm{mg} / \mathrm{kg}$ chlorpromazine (Largactil, Eczacıbaşı, Turkey) and $50 \mathrm{mg} / \mathrm{kg}$ ketamine (Ketalar, Parke Davis, Turkey) combination and were allowed to breathe spontaneously. A mild TBI model, described by Marmarou et al.33 and modified by Ucar et al.34 was applied for head trauma. A lead object weighing 300-gram was allowed to fall freely from a height of 1 meter through a copper tube onto the metal disc over the skull of the rat. In order to reduce mortality thicker metal disc was used during experiments.

\subsection{Collection and storage of brain tissue samples}

All the animals were decapitated 24-hour after trauma, and the brains were carefully removed. Same parts of the brain for the analysis of each parameter obtained from each animal. Brain parts that were used for biochemical analysis were stored at $-80^{\circ} \mathrm{C}$, and brain parts that were used for histological investigations were stored at paraformaldehyde solution.

\subsection{Biochemical analyses}

\subsubsection{Measurement of myeloperoxidase activity in brain tissue}

Myeloperoxidase (MPO), an enzyme that is mainly located in the azurophilic granules of polymorphonuclear leukocytes, is commonly used to demonstrate the accumulation of neutrophils in tissues [35]. Tissue MPO activity was evaluated as previously described [36].

\subsubsection{Chemiluminescence measurements in brain tissue}

Chemiluminescence $(\mathrm{CL})$ is a direct, noninvasive method for the measurement of reactive oxygen radicals that utilizes luminol and lucigenin as enhancer probes. When added to in vitro biological systems, luminol and lucigenin produce high levels of excited products. Excited electrons from these compounds generate radiating light energy or $\mathrm{CL}$ that can be detected by a luminometer. Luminol detects radicals such as hydroxyl ions, hydrogen peroxide, and hydrochloric acid, whereas lucigenin is selective to superoxide anions [37]. Reactive oxygen species (ROS) were numerically measured after the addition of $0.2 \mathrm{mM}$ of enhancers luminol (5-amino-2,3-dihydro- 1,4-phthalazinedione) and lucigenin (bis-N-methylacridinium nitrate) (Sigma Aldrich, USA). Purified luminol-hydrogen peroxide system was used for nitric oxide measurement. For this purpose, potassium carbonate (K2CO3) $(0.4 \mathrm{mM})$, desferal $(60 \mu \mathrm{M}), \mathrm{H} 2 \mathrm{O} 2(4 \mathrm{mM})$ and purified luminol $(3.6 \mathrm{M})$ were added to the tissues in $2 \mathrm{~mL}$ of phosphate-buffered saline (PBS) + HEPES buffer $[38,39]$. Counts were measured at room temperature using a luminometer (Junior LB 9509 luminometer; EG\&G Berthold, Germany), and obtained at 1-minute intervals for 5-minute, the area under the curve was determined, and data expressed as relative light units after counts were normalized to the weight of the brain tissue sample. Results were expressed as relative light units/mg tissue (rlu/mg).

\subsubsection{Enzyme-linked immunosorbent assay measurements}


To determine the levels of interleukin (IL)-1 1 , IL-6, tumor necrosis factor (TNF), IL-10, tumor growth factor (TGF)- $\beta$, PAl-1, caspase 3, and cleaved caspase 3 in the brain tissue, commercial kits (Sunlong Biotech Co. Ltd., China) was used according to the manufacturer's instructions. Supernatants of tissue homogenates were used for measuring the levels of cytokines.

\subsection{Western Blot}

The frozen tissues were weighed and homogenized in ice-cold $10 \mathrm{mM}$ Tris- $\mathrm{HCl}(\mathrm{pH}=7.2)$ buffer containing $1 \mathrm{mM}$ EDTA and protease inhibitors (0.2 mM PMSF, $1 \mu \mathrm{g} / \mathrm{ml}$ leupeptin, $1 \mu \mathrm{M}$ pepsitatin, $10 \mu \mathrm{g} /$ $\mathrm{ml}$ soybean trypsin inhibitors) with Ultraturrax homogenizer. Whole homogenates were used in Western blots. The protein content of the whole homogenate was determined with the Lowry method.40 Mixed with loading buffer (50 mM Tris-HCl pH 6.8, 2\% SDS, 10\% glycerol, 1\% $\beta$-mercaptoethanol, $12.5 \mathrm{mM}$ EDTA, $0.02 \%$ bromophenol blue) samples containing $50 \mathrm{mcg}$ protein have been denatured at $100^{\circ} \mathrm{C}$ for $3-5$ mins and electrophoretically transferred onto nitrocellulose membranes (Schleicher and Schuell, $0.45 \mu \mathrm{m}$, Germany) for 120 min at $80 \mathrm{~V}$. The membranes were blocked with tris-buffered saline containing $1 \%$ bovine serum albumin (Sigma Aldrich, USA) and 0.05\% Tween-20 (Sigma Aldrich, USA) at room temperature for $60 \mathrm{~min}$ and incubated overnight at $4^{\circ} \mathrm{C}$ with antibodies against Matrix metalloproteinase (MMP)-3 (1:500; BIOSS, USA), low density lipoprotein receptor (LDLR)/CREB (1:1000; BIOSS, USA), nuclear factor-KB (NF-KB) (1:500; NOVUS, CO, USA) and $\beta$-actin (1:1000). $\beta$-actin (Sigma Aldrich, USA) was used as an internal control.

The secondary antibodies were purchased from Sigma (St Louis, MO, USA). All chemicals were obtained from Sigma unless stated otherwise. The blots were washed three times with TBS containing $0.05 \%$ Tween-20 (TBS-T) and incubated with alkaline phosphatase-conjugated secondary antibodies for $1 \mathrm{~h}$ at room temperature $\left(20^{\circ} \mathrm{C}\right)$. The antibody-antigen complex was detected with NBT-BCIP(5-bromo-4-chloro-3indolyl phosphate/nitro blue tetrazolium method). The apparent molecular weights of MMP3, CREB, NF$\mathrm{KB}$, and $\beta$-actin $50 \mathrm{kDa}$ are $65 \mathrm{kDa}, 47 \mathrm{kDa}$, respectively. The densitometric analyses were carried out with Image Studio Lite ver5.2 software.

\subsection{Histopathological examinations 3.5.1. Light microscopy}

The brain samples were fixed in the $4 \%$ paraformaldehyde in phosphate buffer $(\mathrm{pH} \mathrm{7.4)}$ for 24-hour at $4^{\circ} \mathrm{C}$. Tissues were then embedded in paraffin and 5 - $\mu \mathrm{m}$-thick coronal sections were created using a rotary microtome. The sections were stained with hematoxylin and eosin stains. Finally, sections were examined under a photomicroscope (Olympus BX51, Japan). The severity of neuronal damage in the cortex was scored semiquantitatively as follows: $0=$ no damage, $1=$ mild damage, $2=$ moderate damage, and $3=$ severe damage. Pyknotic nuclei and intense staining of the shrunken neuronal perikarya were considered in scoring the degree of neuronal degeneration.

\subsubsection{Immunohistochemical investigations}


For immunohistochemical studies, sections were deparaffinized with xylene and rehydrated with $96 \%$ ethanol. Then, the sections were incubated with $3 \%$ hydrogen peroxide solution to inhibit endogenous peroxidase, incubated in the microwave with citrate buffer solution $\left(\mathrm{pH} 6.0,60^{\circ} \mathrm{C}\right)$ for antigen retrieval $(20$ $\mathrm{min})$, and were cooled at room temperature. Then the slides were washed in PBS and treated with protein blocking solution (EXPOSE Rabbit specific HRP/DAB Detection IHC Kit, Abcam, Cambridge, UK). Then the slides were incubated with anti-MMP-3 (1:300, BS-0413R, Bioss Inc, Massachusetts, USA), anti-NF-KB (1:1000, 8242T Cell-Signaling Danvers, MA, USA) and anti-CREB-1 (1:200, BS-0035M, Bioss Inc, Massachusetts, USA) primary antibodies overnight at $4^{\circ} \mathrm{C}$. After washing with PBS, the slides were incubated with biotinylated secondary antibody (Ultra Tek Hrp Anti-Polyvalent, Scytek, USA) for 20 min at room temperature, and then incubated with streptavidin peroxidase for $10 \mathrm{~min}$. Sections were then washed in PBS, exposed with liquid diaminobenzidine (DAB Chromogen/Substrate Kit, Scytek, USA). Slides were counterstained with hematoxylin and were examined with a Olympus BX51 photomicroscope and photographed with a digital camera (Olympus C-5060). Sections were semiquantitatively scored in terms of staining intensity from 0 to 3 (0:none; 1: mild; 2: moderate; 3 : severe).

\subsection{Behavioral tests}

\subsubsection{Novel object recognition test}

The novel object recognition test, which is efficient in evaluating short-term memory, has become a widely used model for the investigation of memory alterations. Alterations in the test results are accepted to be indicative of both hippocampal and cortical lesions [41].

The test is applied as previously described [42]. Difference score (DS; in centisecond), discrimination index ( $\mathrm{Dl}$; in seconds (s)), and recognition index ( $\mathrm{Rl}$; in s) is noted accordingly.

\subsubsection{Y-maze test}

The $Y$ maze is separated by three 120 degree branches from each other and is similar to the T maze. The rat starts at the end of one arm, then chooses between the other two. In this setup, the number of entries into the arms and spontaneous alternation are measured. Spontaneous alternation is measured to assess spatial working memory [43]. Number of entries is countd and spontaneous alternation $\%$ is then calculated with the following formula [43].

\section{Spontaneous alternation $\%=\frac{\# \text { spontaneous alternations }}{\text { total number of arm entries }-2} \times 100$}

\subsubsection{Assessment of Corticospinal Function in Mice with Limb Clasping}

Limb clasping is a functional motor test that measures the loss of corticospinal functions [43]. After 510 seconds of drowning, the subject is videotaped while being held by the tail. Each animal is evaluated 
by two independent observers. It has a score ranging from 0-4 points from normal to immobile. Subjects with a difference of more than 1 point are scored again. The different scores are averaged.

\subsubsection{Modified Bederson neurological examination score}

A 20-point neuro-score was used to assess motor and behavioral deficits [44, 45]. A low score (minimum 0 ) indicates that the animal is awake, active and has normal reflexes. Increasing scores (maximum 20) indicate the occurrence of a neurological disorder. In summary, consciousness, climbing performance on a smooth platform, limb tone, walking, and posture reflexes, circling and response to nociceptive stimuli were evaluated.

\subsection{Statistical analysis}

Data were analyzed using GraphPad Prism 8.0 (GraphPad Software, San Diego, CA, USA) and expressed as means \pm SEM. The effect size was calculated as 0.8 , the standard statistical power $(1-\beta) 0.95$, and the significance level (a) 0.05 with the $G^{*}$ Power programme. Analysis of biochemical and histological data was performed using one-way analysis of variance or Kruskal Wallis test for group comparisons and student T-test was applied for control and TBI group comparisons. In behavior test, Sidak's multiple comparison test was applied. Values of $p<0.05$ were considered to be statistically significant.

\section{Results}

\subsection{Analysis of Biochemical Data}

\subsubsection{Measurements of myeloperoxidase activity, luminol, lucigenin enhanced $\mathrm{CL}$ and nitric oxide release in brain tissues}

A statistically significant increase in MPO values $(p<0.05)$ was observed in the trauma group when compared to the sham group. A statistically significant decrease was observed in TM5441-5 mg $(p<$ $0.01)$, TM5441-10 mg ( $<$ 0.05) group values when compared to the trauma group (Fig. 1A). A statistically significant difference was also found between the treatment groups TM5441-5 mg and TM5484-5 mg $(p<0.05)$.

When NO release was examined, an increase was observed in the trauma group when compared to the sham group (not statistically significant). At the treatment doses of TM5441-5 mg and TM5484-5 mg, NO values were close to the sham group but there was no statistical significance (Fig. 1B). When the treatment groups were compared, a statistically significant difference was found between TM5441-5 mg and TM5441-10 mg $(p<0.05)$.

A statistically significant increase was observed in luminol values in the trauma group when compared to the sham group $(p<0.01)$. Statistically significant decrease was observed in the treatment groups of 
TM5441-5 mg ( $p<0.001)$, TM5441-10 mg ( $<<0.05)$, and TM5484-10 mg $(p<0.05)$ when compared to the sham group. A statistically significant increase was observed in the TM5484-5 mg group when compared to the sham group $(p<0.05)$ (Fig. 1C). When the treatment groups were compared, there was a statistically significant difference among TM5441-5 mg and TM5484-5 mg $(p<0.001)$; TM5441-10 mg and TM5484-5 mg $(p<0.05) ;$ TM5484-5 mg and TM5484-10 mg $(p<0.01)$.

Lucigenin values, which increased statistically significantly in the trauma group when compared to the sham group $(p<0.01)$, were decreased significantly with TM5441-5 mg treatment $(p<0.05)$ (Fig. 1D). An increase was observed in the TM5441-10 mg treatment group when compared to the sham group $(p<$ $0.05)$. When the treatment groups were compared, a significant difference was found between TM5441-5 $\mathrm{mg}$ and TM5441-10 mg ( $<<0.05)$, TM5441-5 mg and TM5484-5 mg $(p<0.05)$.

\subsubsection{Evaluation of PAI-1 level in the brain tissue at the 24th hour after TBI}

A statistically significant increase in the PAl-1 level was observed in the trauma group when compared to the sham group $(p<0.01)$ (Fig. 2). PAl-1 antagonist treatments did not cause any significant change.

\subsubsection{Effects of PAl-1 inhibition on inflammatory cytokines after TBI}

A statistically significant increase was observed in IL-1 $\beta$ level in the trauma group when compared to the sham group $(p<0.05)(F i g .3 A)$. There was a statistically significant difference in IL-1 $\beta$ level between TM5484-5 mg and TM5484-10 mg $(p<0.05)$.

A statistically significant increase was observed in the IL-6 level in the trauma group when compared to the sham group $(p<0.01)$ (Fig. 3B).

There was no statistically significant difference in TNF level between groups (Fig. 3C).

There was no statistically significant difference in IL-10 level in the trauma group when compared to the sham group. A statistically significant increase was found in the TM5484-10 mg group when compared to the sham group $(p<0.05)$ (Fig. 3D). There was a statistically significant difference between TM5484-5 $\mathrm{mg}$ and TM5484-10 mg groups $(p<0.05)$. In TGF- $\beta$ level there was no statistically significant difference was found between groups (Fig. 3E).

\subsubsection{Effects of PAl-1 inhibition on the apoptotic system after TBI}

A statistically significant increase was observed in the caspase-3 activity in the trauma group and the TM5484-10 mg group when compared to the sham group $(p<0.05)$ (Fig. 4A).

Although there was an increase in the cleaved caspase-3 activity in the trauma group when compared to the sham group and a decrease in the treatment groups compared to the trauma group, no statistically 
significant difference was found between the groups (Fig. 4B).

\subsubsection{Measurements of LDLR, MMP3, NF-KB protein levels in brain tissue by Western Blot method}

A statistically significant increase in LDLR / $\beta$ actin level was seen in the trauma group when compared to the sham group ( $p<0.001$, Fig. $5 \mathrm{~A}$ ). A statistically significant decrease was observed in the TM5441-5 mg and $10 \mathrm{mg}$ and TM5484-10 mg groups when compared to the sham group $(\mathrm{p}<0.01-0.001)$. A statistically significant decrease was observed in all treatment groups when compared to the trauma group $(p<0.05-0.001)$. A statistical significance was also found between TM5441-10 mg group and TM5441-5 mg, TM5484- 5, and 10 mg groups ( $<<0.05-0.001)$. A statistically significant difference was also found between TM5484-5 mg and TM5484-10 mg groups ( $p<0.01)$.

There was a statistically significant increase in MMP3 / $\beta$ actin level in the trauma group when compared to the sham group ( $p<0.01$, Fig. 5B). A statistically significant decrease was observed in all treatment groups when compared to the sham and the trauma groups $(p<0.05-0.001)$. A statistically significant difference was also observed between TM5441-10 mg and TM5441-5mg, TM5484-5 and 10 mg groups $(p<0.001)$.

There was a significant increase in the level of NF-KB / $\beta$ actin in the trauma group when compared to the sham group ( $p<0.001$, Fig. $5 C$ ). A statistically significant increase was observed in the TM5484-5 mg group when compared to the sham and the trauma group $(p<0.001)$. A statistically significant increase was observed in the TM5441-5 mg group when compared to the sham group $(p<0.01)$. A significant decrease was observed in the TM5441-10 mg group when compared to the trauma group $(p<0.01)$. There was a significant difference between TM5441-5 mg and TM5441-10 mg and TM5484-5 mg groups $(p<0.01-0.001)$. There was a statistically significant difference between TM5484-5 mg and TM5441-10 mg and TM5484-10 mg groups ( $\mathrm{p}<0.001)$. Western blot bands were shown in Supplementary Fig. 1.

\subsection{HISTOPATHOLOGICAL EVALUATION RESULTS \\ 4.2.1. Light Microscopic Evaluation and Histological Damage Score Results}

Cortex, dentate gyrus (DG), and CA3 regions of the hippocampus were evaluated with a semi-quantitative method in terms of neuronal cell damage with H\&E staining. In the cortex of the rats in the sham group, regular neuropil structure, large nucleus, and prominent nucleolus structures and neurons in uniform morphology were observed (Fig. 6). In the cortex of the rats in the trauma group, neuronal damage, pycnotic cell nuclei, irregularity of the cell structures, and cytoplasmic deterioration were observed when compared with the sham group.

It was observed that this damage was less in the treatment groups. It was observed that the histological damage score $(p<0.001)$, which increased in the trauma group when compared to the sham group, 
decreased in the PAl treatment groups $(p<0.01-0.001)$ (Fig. 7A). Histological damage score for hippocampal DG was increased in the trauma group when compared to the sham group $(p<0.001)$ and decreased in all treatment groups $(p<0.05-0.001)$ (Fig. 7B). The histological damage score for the hippocampal CA3 region was increased in the trauma group when compared to the sham group $(p<0.01)$ and decreased in the TM5441-5 mg group $(p<0.05)$ (Fig. 7C). A statistically significant increase was observed in the histological damage score in the TM5441-10 mg group when compared to the sham group $(\mathrm{p}<0.05)$.

\subsubsection{Results of immunohistochemical examinations}

When the scores of NF-kB immunohistochemistry were evaluated, no statistical significance was found between the groups (Supplementary Fig. 2-3).

\subsection{Behavioral Tests}

\subsubsection{Novel object recognition test}

There was no statistically significant difference in the difference score between the groups in the novel object recognition test (Supplementary Fig. 4A). A statistically significant increase was found in the discrimination index in the sham group when compared to the pre-experiment evaluations (Supplementary Fig. 4B, $p<0.05$ ). Although there was a decrease in the recognition index in the trauma group, there was no statistically significant difference between the groups (Supplementary Fig. 4C).

\subsubsection{Y maze test}

In the $Y$ maze test, a statistically significant decrease was observed in the number of entries in TM5441-5 $\mathrm{mg}$ and TM5484-10 mg treatment groups when compared to pre-procedure values $(p<0.05, p<0.01$; respectively, Sidak' multiple comparison test, Fig. 12A).

A significant decrease was observed in the percentage of spontaneous alternation in the trauma group compared to the sham group ( $p<0.05)$, and no statistically significant difference was found in the other groups (Supplementary Fig. 5).

\subsubsection{Tail suspension test}

A statistically significant increase was observed in the tail suspension test score in the trauma group when compared to the sham group $(p<0.001)$, and a statistically significant decrease was observed in all PAl-1 treatment doses $(p<0.05-p<0.001)$ (Fig. 8).

\subsubsection{Modified Bederson neurological examination score}

The neurological examination score of all experimental animals was 0.

\section{Discussion}


Traumatic brain injury causes microthrombosis and rapid spread of the lesion by disrupting the fibrinolysis mechanisms [16, 46]. In vivo studies demonstrated the formation of microscopic clot areas around the contusion as early as the 1 st hour after the injury $[47,48]$. Appropriate fibrinolysis with plasminogen activators is required to balance clot formation [16]. Anti-inflammatory and neuroprotective activity of the PAl-1 antagonism in neuroinflammatory animal models was previously reported [31, 32]. In this study, TM5441 and TM5484 compounds were used as PAl-1 antagonists in two different doses (5 and $10 \mathrm{mg}$ ) for the first time in a mild TBI animal model. TM5484 has a lower molecular weight than TM5441, and it has been shown to pass through BBB with a higher penetration rate.32 Acute PAI-1 inhibition has been shown to reduce MPO activity, luminol, lucigenin enhanced CL and these results indicated antioxidant and anti-inflammatory properties of these molecules. Histological damage was ameliorated, and corticospinal pathway damage was reversed with PAl-1 antagonism.

In the present study, brain MPO levels, which is an indicator of polymorphonuclear leukocytes (PMNs) significantly increased in the trauma group when compared to the sham group. Similar results were demonstrated in our previous study, where MPO levels were found to be elevated at the 24th hour after TBI [49]. There was a significant decrease in tissue MPO activity with TM5441 treatment indicating a role of PAl-1 in PMN recruitment after brain injury. When the treatment groups were compared among themselves, significantly lower MPO values were found in the TM5441-5 mg group compared to the TM5484-5 mg group.

PAl-1 plays a chemoattractant role for macrophages and PMNs [50]. In previous studies, it has been reported that TM5484 reduces macrophage migration and suppresses microglia activation in the experimental multiple sclerosis model [32]. Activated macrophages, PMNs, and microglia may release NO that its accumulation has been proposed to be associated with some toxic effects [51]. The brain location of this accumulation as well as the machinery of NO production (inducible vs constitutive enzymatic activation) may play an important role for the course of these harmful effects. Villalba et al. [52] reported that after TBI profound increase in endothelial NO production was observed due to increased iNOS expression. In the present study, there was an increase in NO values in the trauma group when compared to the sham group. This increase was leveled off with TM5441-5 and TM5484-5 treatments becoming comparable to the sham group. However, this tendency was not observed with the higher dose of TM5441 (10 mg). Although the increase in NO levels in the trauma group was not significant enough, because this short-lived free radical may be quickly reacting with other molecules/radicals and turning into more stable compounds (eg. Nitrate or nitrite) [53].

It has been previously shown that the levels of luminol and lucigenin, which are markers of oxidative stress and inflammation, increase at the 24th hour after mild TBI [49]. In our study, a significant increase in luminol and lucigenin values was observed in the trauma group when compared to the sham group. It was noted that both parameters significantly decreased after treatments with TM5441 and TM5484 at doses of $5 \mathrm{mg}$. On the other hand, TM5441 but not TM5484 were also effective in reducing luminol levels at the dose of $10 \mathrm{mg}$. These results indicate that after TBI, there was an increase in oxidative stress that somehow involves PAl-1 formation since its antagonism was associated with a reduction in the luminol 
and lucigenin levels. Although there may be various sources of oxidants following the TBI, one plausible candidate is the accumulated PMNs at the site of injury. Our MPO data supported this hypothesis and suggested that reduced oxidative stress following PAl-1 antagonism might be due to a significant reduction in brain MPO activity after the administration of PAI- 1 antagonists especially TM5441. Thus, the significant decrease in inflammatory parameters such as oxidative stress and inflammatory cell accumulation with PAl-1 antagonists was remarkable in terms of the possible therapeutic feature of PAI-1 inhibition.

It has been reported that patients with high PAI-1 levels after TBI have higher neurological damage scores [54]. This situation is thought to be associated with endothelial damage, thrombocyte activation, increased coagulopathy, and venous thrombosis [54]. Consistent with previous studies in the current study, brain PAl-1 level was found to be evaluated at the 24th hour in the trauma group when compared to the sham group. Although the source of increased PAI-1 production after TBI is unclear it can be speculated that PMNs are the unlikely candidates since their tissue level was significantly reduced after TM5441 treatment despite an unaltered PAI-1 level.

In the study of Griemert et al. [16], it was demonstrated that elevated IL-1 $\beta$ and TNF mRNA levels in the pericontusional area were not altered at the 24th hour after TBI with pharmacological inhibition of PAI-1 with PAI-039. On the other hand, Pelisch et al. [32] reported in a multiple sclerosis model that TM5484 was effective in the outcome of neurological/functional recovery as well as in reducing previously increased IL-6, TNF mRNA levels, and decreased IL-10 activity. In a mild TBI model, IL-10 levels after TBI were found to be increased compared to the control group. 55 In the current study, a significant increase was observed in IL-1 $\beta$ and IL- 6 values with trauma, and a decreasing trend was observed in the TM5484-5 mg group. There was no significant difference in the TNF levels between the groups. When we evaluated the anti-inflammatory cytokines, an increasing trend was observed in the trauma. On the other hand, a significant increase in IL-10 level was observed in the TM5484-10 mg group compared to the sham group. It has been shown that IL-1 $\beta$, which is a known proinflammatory cytokine after TBI, binds to the IL1 receptor, causing an increase in NF-kB, cyclooxygenase-2, inducible NO synthase, and reactive oxygen metabolites levels [55]. Although TBI associated production of cytokines is documented by the current as well as by the previous studies, the role of these alterations in TBI pathophysiology is a difficult issue to resolve because of diverse interactions of cytokines with multiple receptors, transcription factors, with each other, the time course of production and the source, etc.

The caspase family is cysteine proteases that regulate many steps of programmed cell death [56, 57]. Caspase-3 breaks down proteins involved in DNA repair $[58,59]$. It is known that cytoskeletal proteins such as actin and spectrin are degraded by caspase-3 activation $[60,61]$. Cleaved caspase-3 is the active form of caspase-3, and it was reported that its increased values were associated with the unfavorable outcome [62]. It has been shown that there was an increase in caspase-3 enzyme activity at the 24th hour after TBI [49, 56]. Griemert et al. [17]. showed that apoptotic cells decreased at 24 hours after TBI following PAl-1 antagonist treatment. In our study, we showed that there was a significant increase in caspase-3 values and that the cleaved caspase-3 level tended to increase after TBI. Treatment with PAI-1

Page 13/28 
antagonists, on the other hand, resulted in a decrease in cleaved caspase-3 levels indicating decreased apoptotic activity.

Matrix metalloproteinases are the second important proteolytic system in the mammalian brain and although they may play a role in the protection of neurological functions, they can also cause damage to the BBB. Under ischemic stress, proteolytically active tPA causes MMP-3 induction from endothelial cells through the LDLR and NF-KB pathways [63]. On the other hand, when PAl-1 complexes with TPA or uPA these complexes are then taken into the cell and cleaned by binding to the LDLR from the circulation [6466]. Pharmacological inhibition of previously increased MMP-3 significantly attenuated neurovascular permeability and improved neurological functions [67]. Similarly, it was found that tPA-PAI complex and MMP-3 levels increased in the CSF of patients with TBI and were associated with a negative neurological outcome. In another study, it was found that the levels of albumin and MMP-3, as indicators of cerebrovascular damage, were increased in the brain tissue of patients with head trauma [68]. It was reported that tPA activity increased by $30 \%$ within 1-3 hours after trauma and returned to its baseline value at the 24th hour [68]. These studies indicate the importance of biological steps after PAI-1-tPA complex formation and suggest a role of LDLR and MMP in TBI pathophysiology.

In our study, western blotting demonstrated an increase in the LDLR, NF-KB, and MMP3 protein levels in the trauma group compared to the sham group. LDLR and MMP3 levels decreased and NF-KB levels increased in the treatment groups. PAI- 1 inhibition may have reduced the possible effects of MMP and LDLR activations on inflammatory pathways. Mettang et al.[69] evaluated the inhibition of NF-KB in the TBI model and reported that the inflammatory response was enhanced, neuronal apoptosis and brain damage was increased, and functional outcomes were negatively affected. The protective role of NF-KB in neurons includes the antiapoptotic effects mediated by inducing endogenous caspase inhibitors or by triggering the expression of antioxidant genes [70, 71], as NF-KB transcription factors regulate a wide range of survival-promoting target genes [72]. In our study, treatment with PAl-1 antagonists increased $\mathrm{NF-KB}$ levels that are compatible with this scenario. As seen in western blot results, immunohistochemical expression of NF-KB was also increased in the trauma group, and this increase was higher in the PAI antagonist group. A further increase in NF-KB levels in the treatment groups may indicate the activation of some protective mechanisms following brain damage.

The histological damage score significantly increased in the cortex, hippocampal DG, and CA3 regions and decreased in all treatment groups except CA3. In the CA3 region, a significant reduction was only detected in the TM5441-5 mg group. In a TBI model, Griemert et al.16 evaluated neuronal loss in the DG with TUNEL staining and reported that PAI-1 inhibition had a positive impact. This was thought to be due to improved cerebral perfusion. Taken together our biochemical and histological data support each other and suggest that reduced neutrophil infiltration, and oxidative stress observed with PAl-1 inhibition, and possible modulation of the peptidase/protease pathway positively affect cell morphology and histological damage scores. 
In the current study, changes in cognitive functions and neurological status before and 24-hour after TBI were evaluated with Y-maze, novel object recognition, tail suspension, and neurological tests for the first time in the literature. In the novel object recognition test, a decreased tendency in the discrimination and recognition indexes were observed in the trauma group when compared to the sham group. In most of the treatment groups, values were close to the sham. In the $Y$ maze test, the number of entries was decreased in the trauma group when compared to pre-procedure values in all groups, and this response was found to be compatible with the freezing responses observed during the experiment. Similarly, the spontaneous alternation percentages were lower in the trauma group when compared to the pre-procedure values. Additionally, a significant decrease was observed in the trauma group when compared to the sham group, and an increasing trend was noted in all treatment groups.

Previously a loss in locomotor function was shown on the 11th day after mild TBI [55]. In the current study, the application of the tail suspension test to evaluate corticospinal function demonstrated a significant increase in damage score after TBI and elevated damage score significantly decreased in all treatment groups. Overall, our behavioral tests represented that mild TBI might alter not all but some of the patterns of actions performed by the whole animal indicating that inflammation at the 24th hour of trauma was associated with some cognitive and behavioral changes. Furthermore, it was conceivable to suggest that while PAI-1 inhibition was effective in reducing PMN infiltration, oxidative stress, histological damage score, it was also associated with better functional outcomes.

As seen in any laboratory study, there are also limitations in this study. The neuroprotective efficacy of PAl antagonist treatment at different doses and different treatment durations with different TBI models can be studied more comprehensively. Especially in learning-memory tests, the number of subjects is insufficient to show possible differences among groups because the data does not show a normal distribution. The changes in other periods could also be evaluated. In this study, the aim of applying PAI antagonist treatment immediately after TBI is to reach the effective blood concentration immediately and try to reduce the effects of the injury mechanisms. However, since it is not possible to provide treatment immediately after TBI in the clinical settings, the effectiveness of the treatment can be examined in a more realistic model by applying PAI antagonist treatment after a certain time after TBI.

Besides, in some studies, PAI-1 has also been reported to have a neuroprotective effect through the mitogen-activated protein kinase/extracellular signal-mediated kinase signaling pathway [73]. It has been reported that intracerebral PAI-1 administration is also neuroprotective in the neonatal cerebral hypoxia model [74]. For this reason, it should be kept in mind in future studies that PAl-1 may show a dual effect in different conditions.

\section{Conclusion}

In this study, PAl-1 inhibition was achieved after TBI with TM5441 and TM5484 PAl-1 antagonists for the first time, and their effects on inflammation, oxidative stress, apoptosis, neuronal damage, and functional outcomes after TBI were investigated. PAI-1 inhibition has been shown to reduce oxidative stress and 
neuronal damage, and improve damage to the corticospinal pathway. Examining the effects of PAl-1 inhibition on both pathophysiological and functional results in TBI and obtaining promising results would shed light on future clinical studies.

\section{Abbreviations}

CL: Chemiluminescence

DG:Dentate gyrus

DI:Discrimination index

DS:Difference score

IL: Interleukin

LDLR: Low density lipoprotein receptor

MMP3: Matrix metalloproteinase-3

MPO: Myeloperoxidase

NF-kB: Nuclear factor-kB

NO: Nitric oxide

PAl-1: Plasminogen activator inhibitor-1

PBS: Phosphate-buffered saline

PMNs: Polymorphonuclear leukocytes

Rl: Recognition index

ROM: Reactive oxygen metabolites

ROS: Reactive oxygen species

TBI: Traumatic brain injury

TGF: Tumor growth factor

TNF: Tumor necrosis factor

tPA: tissue plasminogen activator 


\section{Declarations}

Ethics approval and consent to participate: All experimental procedures used in this investigation were reviewed and approved by the Marmara University Animal Care and Use Committee (12.2019.mar).

Consent for publication: All authors consent to the publication of the manuscript in Inflammation.

Availability of data and materials: The data and materials are available on request.

Competing interests: The authors report no competing interests.

Funding: This work was supported by Marmara University Scientific Research Projects Coordination Unit.

Authors' contributions: Pınar Kuru Bektaşoğlu: Conceptualization, Methodology, Data curation, WritingOriginal draft preparation, Writing- Reviewing and Editing. Turkan Koyuncuoglu: Data curation. Selin Akbulut: Data curation. Dilek Akakın: Data curation, Writing- Reviewing and Editing. İrem Peker Eyüboğlu: Data curation. Can Erzik: Data curation. Meral Yüksel: Data curation, Writing- Reviewing and Editing. Hizir Kurtel: Visualization, Investigation, Supervision, Writing- Reviewing and Editing.

Acknowledgements: We would like to thank Prof. Toshio Miyata and Takashi Dan for their generous support in sending us PAl antagonists.

\section{References}

1. Menon, D. K., K. Schwab, D. W. Wright, A. I. Maas. Demographics and Clinical Assessment Working Group of the International and Interagency Initiative toward Common Data Elements for Research on Traumatic Brain Injury and Psychological Health. Position statement: definition of traumatic brain injury. Arch Phys Med Rehabil. 2010;91(11):1637-1640.

2. Bomyea, J., A. J. Lang, and P. P. Schnurr. 2017. TBI and Treatment Response in a Randomized Trial of Acceptance and Commitment Therapy. J Head Trauma Rehabil 32 (5): E35-E43.

3. Ghajar, J. 2000. Traumatic brain injury. Lancet 356: 923-929.

4. Liu, B., G. Zhang, S. Cui, and G. Du. 2020. Inhibition of RNF6 alleviates traumatic brain injury by suppressing STAT3 signaling in rats. Brain Behav 10 (12): e01847.

5. Maas, A. I. R., D. K. Menon, P. D. Adelson, N. Andelic, M. J. Bell, and A. Belli, et al. 2017. Traumatic brain injury: integrated approaches to improve prevention, clinical care, and research. Lancet Neurol 16 (12): 987-1048.

6. Miles, S. R., J. M. Harik, N. E. Hundt, J. Mignogna, N. J. Pastorek, and K. E. Thompson, et al. 2017. Delivery of mental health treatment to combat veterans with psychiatric diagnoses and TBI histories. PLoS One 12 (9): e0184265.

7. Marshall, C. A., E. Nalder, H. Colquhoun, E. Lenton, M. Hansen, and D. R. Dawson, et al. 2019. Interventions to address burden among family caregivers of persons aging with TBI: A scoping 
review. Brain Inj 33 (3): 255-265.

8. Zaninotto, A. L., M. M. El-Hagrassy, J. R. Green, M. Babo, V. M. Paglioni, and G. G. Benute, et al. 2019. Transcranial direct current stimulation (tDCS) effects on traumatic brain injury (TBI) recovery: A systematic review. Dement Neuropsychol 13 (2): 172-179.

9. Gardner, R. C., A. L. Byers, D. E. Barnes, Y. Li, J. Boscardin, and K. Yaffe. 2018. Mild TBI and risk of Parkinson disease: A Chronic Effects of Neurotrauma Consortium Study. Neurology 90 (20): e1771e1779.

10. Feigin, V. L., A. Theadom, S. Barker-Collo, N. J. Starkey, K. McPherson, and M. Kahan, et al. 2013. Incidence of traumatic brain injury in New Zealand: a population-based study. Lancet Neurol 12 (1): 53-64.

11. Prince, C., and M. E. Bruhns. 2017. Evaluation and Treatment of Mild Traumatic Brain Injury: The Role of Neuropsychology. Brain Sci 7 (8): 105.

12. Biegon, A., P. A. Fry, C. M. Paden, A. Alexandrovich, J. Tsenter, and E. Shohami. 2004. Dynamic changes in N-methyl-D-aspartate receptors after closed head injury in mice: implications for treatment of neurological and cognitive deficits. Proc Natl Acad Sci USA 101: 5117-5122.

13. Sullivan, P. G., J. N. Keller, M. P. Mattson, and S. W. Scheff. 1998. Traumatic brain injury alters synaptic homeostasis: implications for impaired mitochondrial and transport function. $J$ Neurotrauma 15: 789-798.

14. Salehi, A., J. H. Zhang, and A. Obenaus. 2017. Response of the cerebral vasculature following traumatic brain injury. J Cereb Blood Flow Metab 37 (7): 2320-2339.

15. Woodcock, T., and M. C. Morganti-Kossmann. 2013. The role of markers of inflammation in traumatic brain injury. Front Neurol 4: 18.

16. Griemert, E. V., S. M. Schwarzmaier, R. Hummel, C. Gölz, D. Yang, and W. Neuhaus, et al. 2019a. Plasminogen activator inhibitor-1 augments damage by impairing fibrinolysis after traumatic brain injury. Ann Neurol 85 (5): 667-680.

17. Griemert, E. V., K. Recarte Pelz, K. Engelhard, M. K. Schäfer, and S. C. Thal. 2019b. PAl-1 but Not PAl-2 Gene Deficiency Attenuates Ischemic Brain Injury After Experimental Stroke. Trans/ Stroke Res 10 (4): 372-380.

18. Brogren, H., L. Karlsson, M. Andersson, L. Wang, D. Erlinge, and S. Jern. 2004. Platelets synthesize large amounts of active plasminogen activator inhibitor 1. Blood 104 (13): 3943-3948.

19. Huang, J., M. Sabater-Lleal, F. W. Asselbergs, D. Tregouet, S. Y. Shin, and J. Ding, et al. 2012. Genomewide association study for circulating levels of PAl-1 provides novel insights into its regulation. Blood 120 (24): 4873-4881.

20. Hultman, K., F. Blomstrand, M. Nilsson, U. Wilhelmsson, K. Malmgren, and M. Pekny, et al. 2010. Expression of plasminogen activator inhibitor-1 and protease nexin-1 in human astrocytes: Response to injury-related factors. J Neurosci Res 88 (11): 2441-2449.

21. Simpson, A. J., N. A. Booth, N. R. Moore, and B. Bennett. 1991. Distribution of plasminogen activator inhibitor (PAI-1) in tissues. J Clin Pathol 44 (2): 139-143. 
22. Giesen, P. L., and Y. Nemerson. 2000. Tissue factor on the loose. Semin Thromb Hemost 26 (4): 379384.

23. Emeis, J. J. 1992. Regulation of the acute release of tissue-type plasminogen activator from the endothelium by coagulation activation products. Ann N Y Acad Sci 667: 249-258.

24. Gorlatova, N. V., J. M. Cale, H. Elokdah, D. Li, K. Fan, and M. Warnock, et al. 2007. Mechanism of inactivation of plasminogen activator inhibitor-1 by a small molecule inhibitor. J Biol Chem 282 (12): 9288-9296.

25. Lawrence, D. A., S. T. Olson, S. Muhammad, D. E. Day, J. O. Kvassman, and D. Ginsburg, et al. 2000. Partitioning of serpin-proteinase reactions between stable inhibition and substrate cleavage is regulated by the rate of serpin reactive center loop insertion into beta-sheet A. J Biol Chem 275 (8): $5839-5844$.

26. Kang, S., T. Tanaka, H. Inoue, C. Ono, S. Hashimoto, and Y. Kioi, et al. 2020. IL-6 trans-signaling induces plasminogen activator inhibitor-1 from vascular endothelial cells in cytokine release syndrome. Proc Natl Acad Sci U S A 117 (36): 22351-22356.

27. van der Poll, T., M. Levi, H. R. Büller, S. J. van Deventer, J. P. de Boer, and C. E. Hack, et al. 1991. Fibrinolytic response to tumor necrosis factor in healthy subjects. J Exp Med 174 (3): 729-732.

28. Lip, G. Y., A. D. Blann, I. S. Farooqi, J. Zarifis, G. Sagar, and D. G. Beevers. 2002. Sequential alterations in haemorheology, endothelial dysfunction, platelet activation and thrombogenesis in relation to prognosis following acute stroke: The West Birmingham Stroke Project. Blood Coagul Fibrinolysis 13 (4): 339-347.

29. Tuttolomondo, A., A. Pinto, S. Corrao, D. Di Raimondo, P. Fernandez, and R. Di Sciacca, et al. 2009. Immuno-inflammatory and thrombotic/fibrinolytic variables associated with acute ischemic stroke diagnosis. Atherosclerosis 203 (2): 503-508.

30. Beuth, W., M. Kotschy, H. A. Kasprzak, D. Rosc, P. Osinski, and M. Sniegocki. 1996. [Tissue plasminogen activator (T-PA) and tissue plasminogen activator inhibitor (PAl-1) in patients after head injury]. Neurol Neurochir Po/30 (3): 427-434.

31. Chan, S. L., N. Bishop, Z. Li, and M. J. Cipolla. 2018. Inhibition of PAl (Plasminogen Activator Inhibitor)-1 Improves Brain Collateral Perfusion and Injury After Acute Ischemic Stroke in Aged Hypertensive Rats. Stroke 49 (8): 1969-1976.

32. Pelisch, N., T. Dan, A. Ichimura, H. Sekiguchi, D. E. Vaughan, C. van Ypersele de Strihou, and T. Miyata. 2015. Plasminogen Activator Inhibitor-1 Antagonist TM5484 Attenuates Demyelination and Axonal Degeneration in a Mice Model of Multiple Sclerosis. PLoS One 10 (4): e0124510.

33. Marmarou, A., M. A. Foda, W. van den Brink, J. Campbell, H. Kita, and K. Demetriadou. 1994. A new model of diffuse brain injury in rats. Part I: Pathophysiology and biomechanics. J Neurosurg 80 (2): 291-300.

34. Ucar, T., G. Tanriover, I. Gurer, M. Z. Onal, and S. Kazan. 2006. Modified experimental mild traumatic brain injury model. J Trauma 60 (3): 558-565. 
35. Tuğtepe, H., G. Sener, N. K. Biyikli, M. Yüksel, S. Cetinel, and N. Gedik, et al. 2007. The protective effect of oxytocin on renal ischemia/reperfusion injury in rats. Regul Pept 140 (3): 101-108.

36. Bradley, P. P., D. A. Priebat, R. D. Christensen, and G. Rothstein. 1982. Measurement of cutaneous inflammation: estimation of neutrophil content with an enzyme marker. $J$ Invest Dermatol 78 (3): 206-209.

37. Deshpande, S. S. 2001. Principles and applications of luminescence spectroscopy. Crit Rev Food Sci Nutr 41 (3): 155-224.

38. Kikuchi, K., T. Nagano, H. Hayakawa, Y. Hirata, and M. Hirobe. 1993a. Real time measurement of nitric oxide produced ex vivo by luminol-H2O2 chemiluminescence method. J Biol Chem 268 (31): 23106-23110.

39. Kikuchi, K., T. Nagano, H. Hayakawa, Y. Hirata, and M. Hirobe. 1993b. Detection of nitric oxide production from a perfused organ by a luminol-H2O2 system. Anal Chem 65 (13): 1794-1799.

40. Lowry, O. H., N. J. Rosebrough, A. L. Farr, and R. J. Randall. 1951. Protein measurement with the Folin phenol reagent. J Biol Chem 193 (1): 265-275.

41. Antunes, M., and G. Biala. 2012. The novel object recognition memory: neurobiology, test procedure, and its modifications. Cogn Process 13 (2): 93-110.

42. Lueptow, L. M. Novel Object Recognition Test for the Investigation of Learning and Memory in Mice. J Vis Exp. 2017;(126):55718. doi:10.3791/55718.

43. Miedel, C. J., J. M. Patton, A. N. Miedel, E. S. Miedel, and J. M. Levenson. Assessment of Spontaneous Alternation, Novel Object Recognition and Limb Clasping in Transgenic Mouse Models of Amyloid- $\beta$ and Tau Neuropathology. J Vis Exp. 2017;(123):55523.

44. Bederson, J. B., L. H. Pitts, M. Tsuji, M. C. Nishimura, R. L. Davis, and H. Bartkowski. 1986. Rat middle cerebral artery occlusion: evaluation of the model and development of a neurologic examination. Stroke 17 (3): 472-476.

45. Toklu, H. Z., M. K. Uysal, L. Kabasakal, S. Sirvanci, F. Ercan, and M. Kaya. 2009. The effects of riluzole on neurological, brain biochemical, and histological changes in early and late term of sepsis in rats. J Surg Res 152 (2): 238-248.

46. Huber, A., A. Dorn, A. Witzmann, and J. Cervos-Navarro. 1993. Microthrombi formation after severe head trauma. Int J Legal Med 106 (3): 152-155.

47. Dietrich, W. D., O. Alonso, and M. Halley. 1994. Early microvascular and neuronal consequences of traumatic brain injury: a light and electron microscopic study in rats. J Neurotrauma 11 (3): 289301.

48. Schwarzmaier, S. M., S. W. Kim, R. Trabold, and N. Plesnila. 2010. Temporal profile of thrombogenesis in the cerebral microcirculation after traumatic brain injury in mice. J Neurotrauma 27 (1): 121-130.

49. Demir, D., P. Kuru Bektaşoğlu, T. Koyuncuoğlu, C. Kandemir, D. Akakın, and M. Yüksel, et al. 2019. Neuroprotective effects of mildronate in a rat model of traumatic brain injury. Injury 50 (10): 15861592. 
50. Ichimura, A., S. Matsumoto, S. Suzuki, T. Dan, S. Yamaki, and Y. Sato, et al. 2013. A small molecule inhibitor to plasminogen activator inhibitor 1 inhibits macrophage migration. Arterioscler Thromb Vasc Biol 33 (5): 935-942.

51. Redford, E. J., R. Kapoor, and K. J. Smith. 1997. Nitric oxide donors reversibly block axonal conduction: demyelinated axons are especially susceptible. Brain 120 (Pt 12): 2149-2157.

52. Villalba, N., S. K. Sonkusare, T. A. Longden, T. L. Tran, A. M. Sackheim, and M. T. Nelson, et al. 2014. Traumatic brain injury disrupts cerebrovascular tone through endothelial inducible nitric oxide synthase expression and nitric oxide gain of function. J Am Heart Assoc 3 (6): e001474.

53. Tisdall, M. M., K. Rejdak, N. D. Kitchen, M. Smith, and A. Petzold. 2013. The prognostic value of brain extracellular fluid nitric oxide metabolites after traumatic brain injury. Neurocrit Care 19 (1): 65-68.

54. Condron, M., S. Rowell, E. Dewey, T. Anderson, L. Lealiiee, and D. Farrell, et al. 2018. The procoagulant molecule plasminogen activator inhibitor-1 is associated with injury severity and shock in patients with and without traumatic brain injury. J Trauma Acute Care Surg 85 (5): 888-893.

55. Perez-Polo, J. R., H. C. Rea, K. M. Johnson, M. A. Parsley, G. C. Unabia, and G. Xu, et al. 2013. Inflammatory consequences in a rodent model of mild traumatic brain injury. J Neurotrauma 30 (9): 727-740.

56. Clark, R. S., P. M. Kochanek, S. C. Watkins, M. Chen, C. E. Dixon, and N. A. Seidberg, et al. 2000. Caspase-3 mediated neuronal death after traumatic brain injury in rats. J Neurochem 74 (2): $740-$ 753.

57. Saunders, J. W. 1966. Jr. Death in embryonic systems. Science 154: 604-612.

58. Lazebnik, Y. A., S. H. Kaufmann, S. Desnoyers, G. G. Poirier, and W. C. Earnshaw. 1994. Cleavage of poly(ADP-ribose) polymerase by a proteinase with properties like ICE. Nature 371 (6495): 346-347.

59. Song, Q., S. P. Lees-Miller, S. Kumar, Z. Zhang, D. W. Chan, and G. C. Smith, et al. 1996. DNAdependent protein kinase catalytic subunit: a target for an ICE-like protease in apoptosis. EMBO J15 (13): 3238-3246.

60. Kothakota, S., T. Azuma, C. Reinhard, A. Klippel, J. Tang, and K. Chu, et al. 1997. Caspase-3-generated fragment of gelsolin: effector of morphological change in apoptosis. Science 278 (5336): 294-298.

61. Pike, B. R., X. Zhao, J. K. Newcomb, R. M. Posmantur, K. K. Wang, and R. L. Hayes. 1998. Regional calpain and caspase-3 proteolysis of alpha-spectrin after traumatic brain injury. Neuroreport 9 (11): 2437-2442.

62. Liu, P. F., Y. C. Hu, B. H. Kang, Y. K. Tseng, P. C. Wu, and C. C. Liang, et al. 2017. Expression levels of cleaved caspase-3 and caspase-3 in tumorigenesis and prognosis of oral tongue squamous cell carcinoma. PLoS One 12 (7): e0180620.

63. Suzuki, Y., N. Nagai, K. Yamakawa, J. Kawakami, H. R. Lijnen, and K. Umemura. 2009. Tissue-type plasminogen activator (t-PA) induces stromelysin-1 (MMP-3) in endothelial cells through activation of lipoprotein receptor-related protein. Blood 114 (15): 3352-3358.

64. Candelario-Jalil, E., Y. Yang, and G. A. Rosenberg. 2009. Diverse roles of matrix metalloproteinases and tissue inhibitors of metalloproteinases in neuroinflammation and cerebral ischemia. 
Neuroscience 158 (3): 983-994.

65. Herz, J. 2001. The LDL receptor gene family: (un)expected signal transducers in the brain. Neuron 29 (3): 571-581.

66. Lillis, A. P., L. B. Van Duyn, J. E. Murphy-Ullrich, and D. K. Strickland. 2008. LDL receptor-related protein 1: unique tissue-specific functions revealed by selective gene knockout studies. Physiol Rev 88 (3): 887-918.

67. Sashindranath, M., E. Sales, M. Daglas, R. Freeman, A. L. Samson, and E. J. Cops, et al. 2012. The tissue-type plasminogen activator-plasminogen activator inhibitor 1 complex promotes neurovascular injury in brain trauma: evidence from mice and humans. Brain 135 (Pt 11): 32513264.

68. Sashindranath, M., A. L. Samson, C. E. Downes, P. J. Crack, A. J. Lawrence, and Q. X. Li, et al. 2011. Compartment- and context-specific changes in tissue-type plasminogen activator (tPA) activity following brain injury and pharmacological stimulation. Lab Invest 91 (7): 1079-1091.

69. Mettang, M., S. N. Reichel, M. Lattke, A. Palmer, A. Abaei, and V. Rasche, et al. 2018. IKK2/NF-KB signaling protects neurons after traumatic brain injury. FASEB J 32 (4): 1916-1932.

70. Qin, Z. H., L. Y. Tao, and X. Chen. 2007. Dual roles of NF-kappaB in cell survival and implications of NF-kappaB inhibitors in neuroprotective therapy. Acta Pharmacol Sin 28 (12): 1859-1872.

71. Sanz, O., L. Acarin, B. González, and B. Castellano. 2002. NF-kappaB and IkappaBalpha expression following traumatic brain injury to the immature rat brain. J Neurosci Res 67 (6): 772-780.

72. Yang, L., L. Y. Tao, and X. P. Chen. 2007. Roles of NF-kappaB in central nervous system damage and repair. Neurosci Bull 23 (5): 307-313.

73. Soeda, S., S. Koyanagi, Y. Kuramoto, M. Kimura, M. Oda, and T. Kozako, et al. 2008. Anti-apoptotic roles of plasminogen activator inhibitor-1 as a neurotrophic factor in the central nervous system. Thromb Haemost 100 (6): 1014-1020.

74. Yang, D., N. Nemkul, A. Shereen, A. Jone, R. S. Dunn, and D. A. Lawrence, et al. 2009. Therapeutic administration of plasminogen activator inhibitor-1 prevents hypoxic-ischemic brain injury in newborns. J Neurosci 29 (27): 8669-8674.

\section{Figures}


$\square$ TM5441 $(\mathrm{mg} / \mathrm{kg})$
TM5584 $(\mathrm{mg} / \mathrm{kg})$

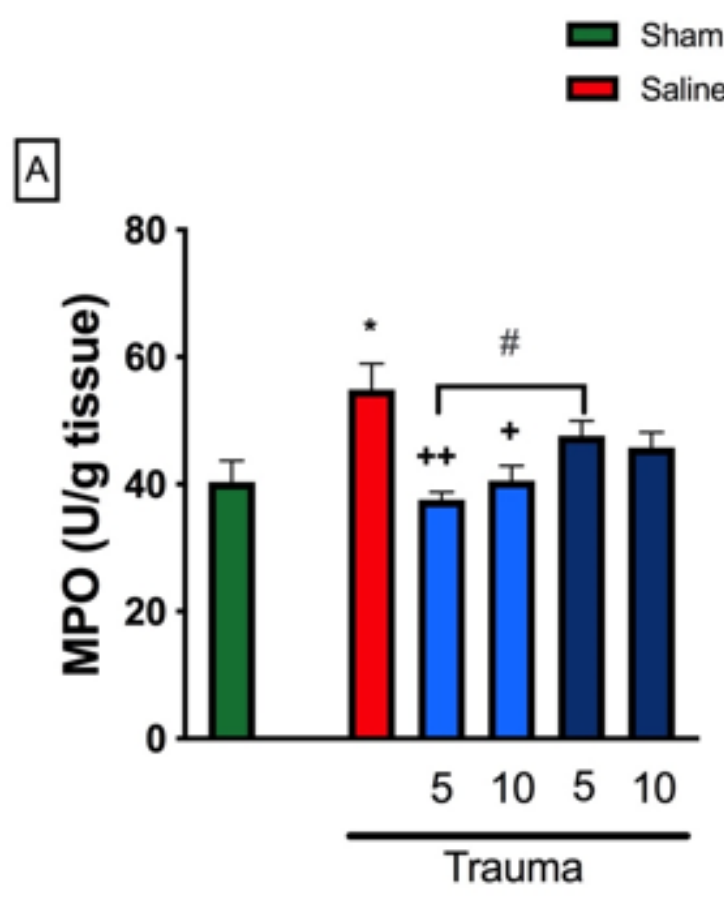

A

C

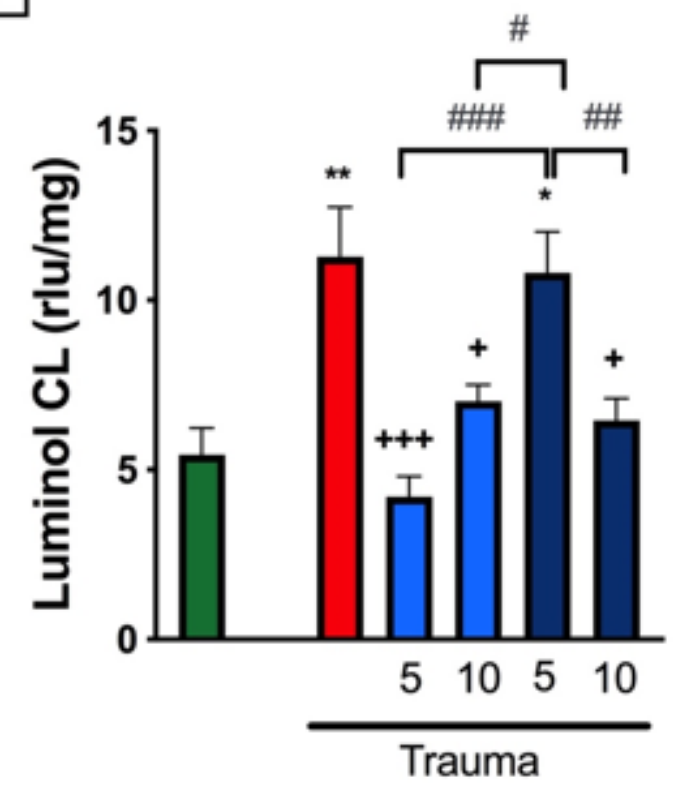

B

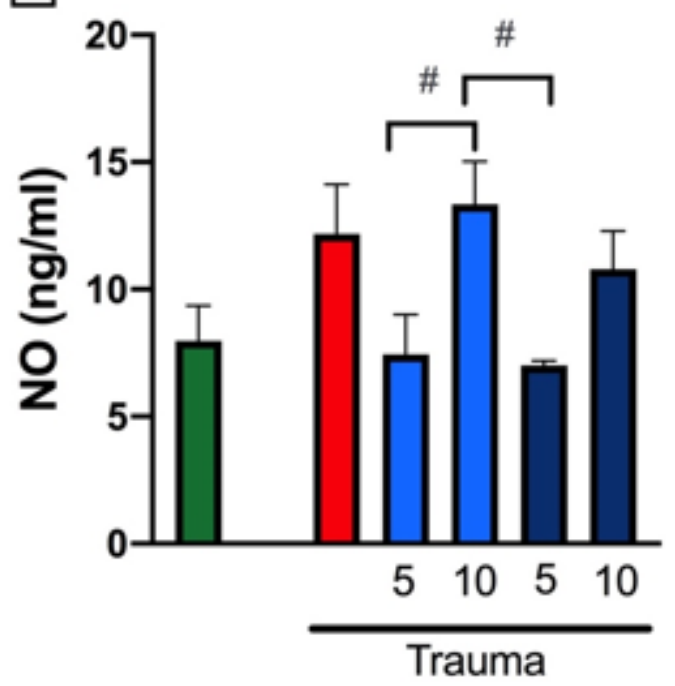

D

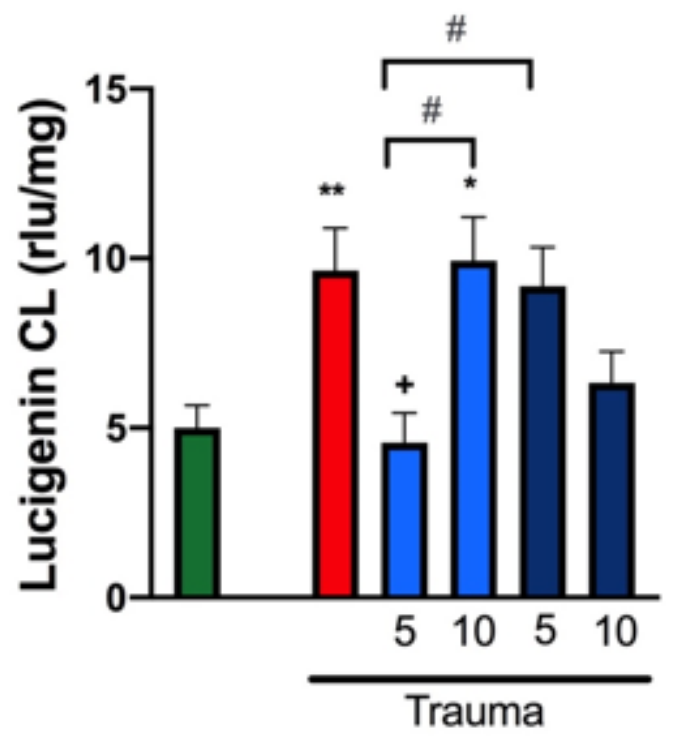

Figure 1

A) Myeloperoxidase (MPO), B) Nitric Oxide (NO), C) Luminol and D) Lusigenin values of experimental groups (According to the sham group $\star \star \star$ : $p<0.001$; according to the trauma group $+: p<0.05,+++$ : $p$ $<0.001$, among treatment groups: \# \#: $\mathrm{p}<0.01$, \# \# \# $\mathrm{p}<0.001)$. 


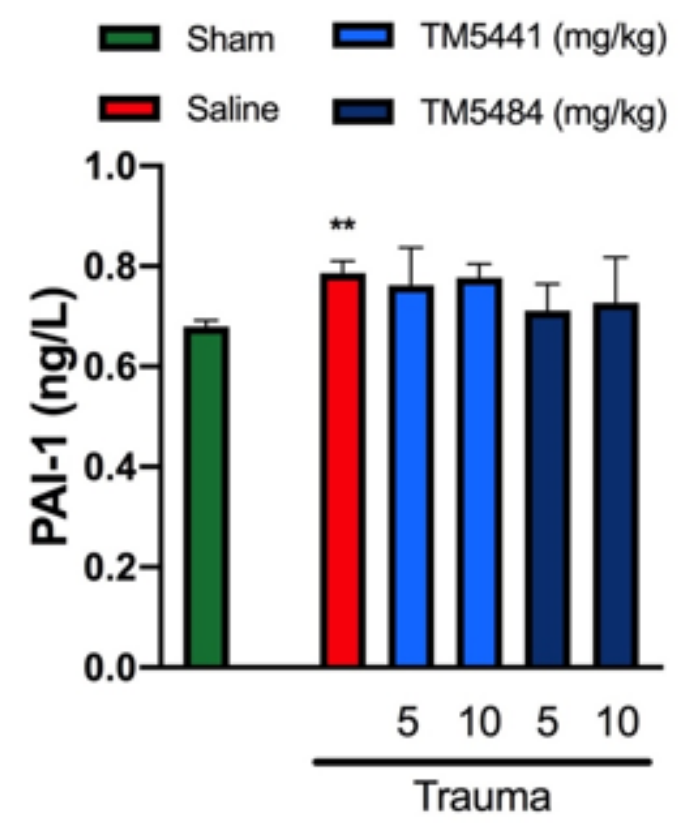

Figure 2

PAl levels of experimental groups (According to the sham group $* *$ : $p<0.01$ ).

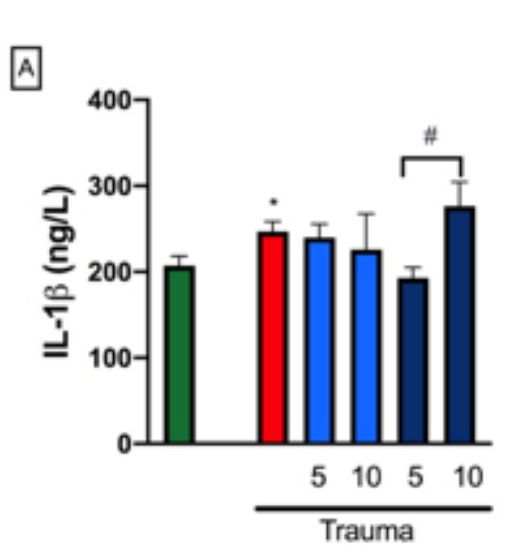

D

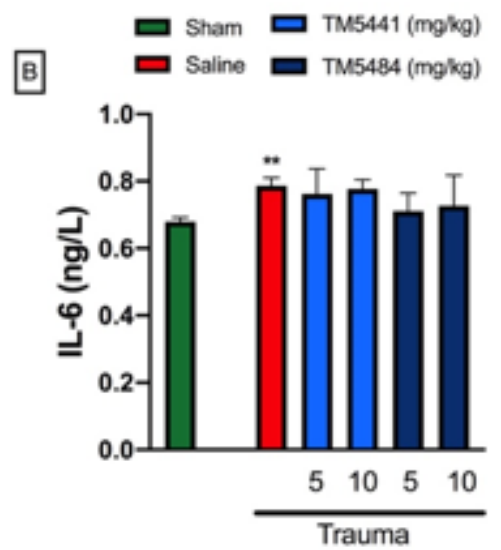

国

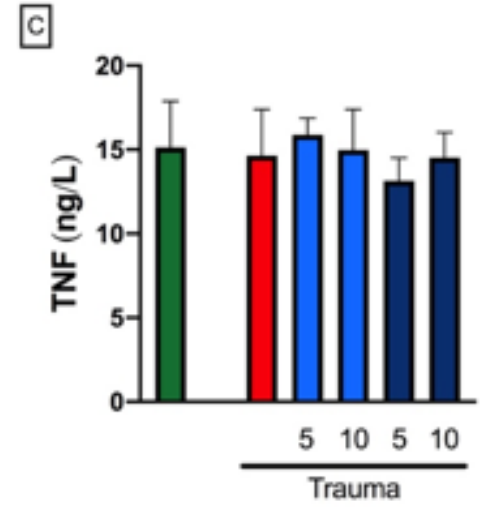

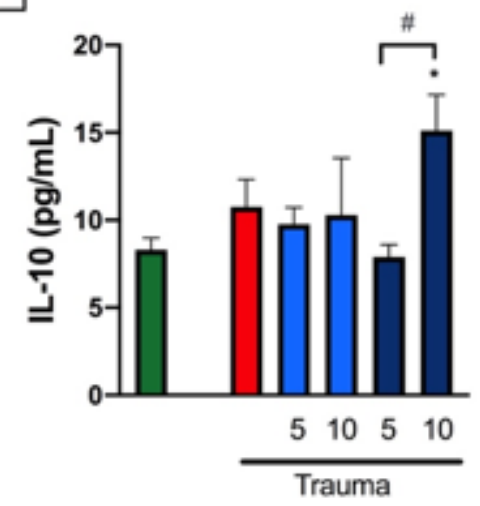

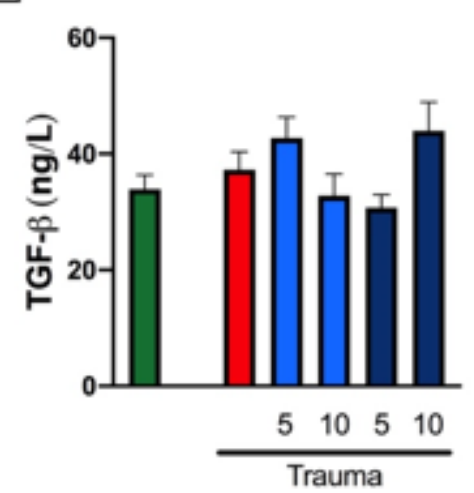

Figure 3 
A) IL-1 $\beta$, B) IL-6, C) TNF, D) IL-10, and D) TGF- $\beta$ levels of experimental groups (According to the sham group $*$ : $p<0.05, * *: p<0.01$; among treatment groups: $\#$ : $p<0.05$ )

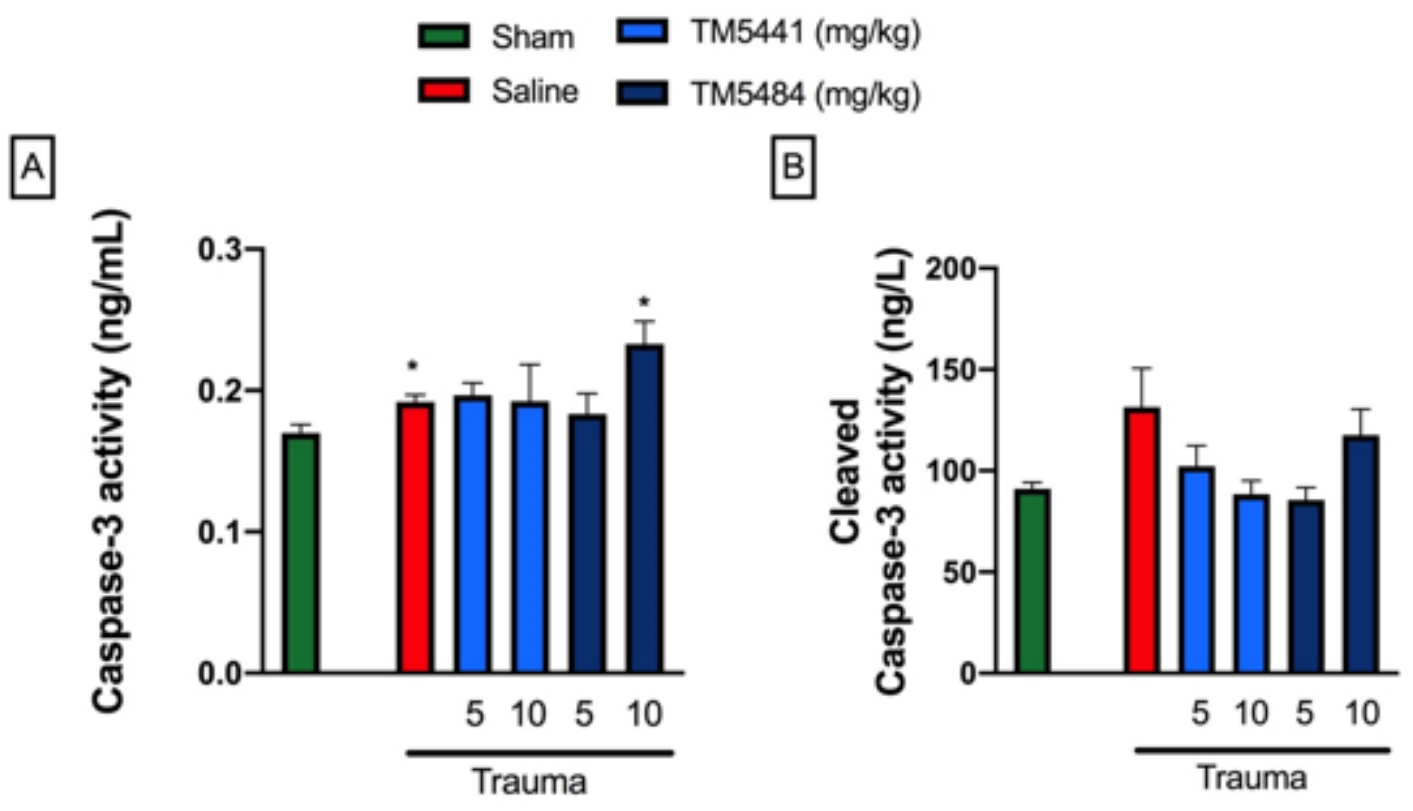

Figure 4. A) Caspase-3 and B) Cleaved caspase-3 levels of experimental groups (According to the sham group *: $\mathrm{p}<0.05$ ).

Figure 4

A) Caspase-3 and B) Cleaved caspase-3 levels of experimental groups (According to the sham group *: $p$ $<0.05)$.

A

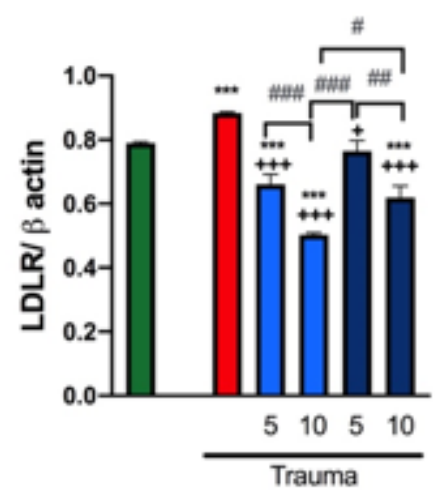

$\square$ Sham TM5441 (mg/kg)

$\square$ Saline TM5484 (mg/kg)

目

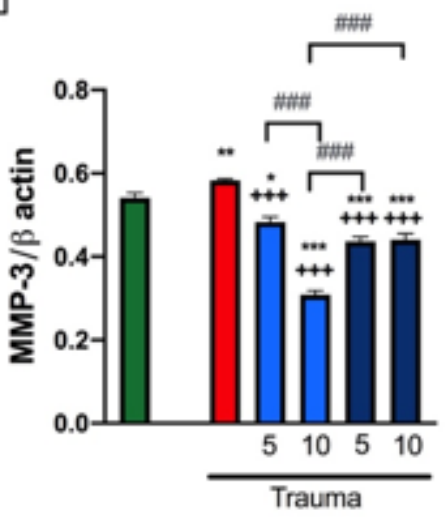

c

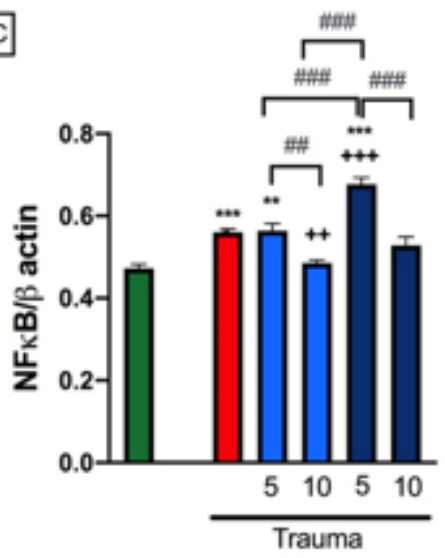

Figure 5 
A) LDLR/ $\beta$ actin, B) MMP3/ $\beta$ actin ve $C$ ) NF-KB/ $\beta$ actin levels of experimental groups (According to the sham group $*: p<0.05, * *: p<0.01, * \star *: p<0.001$; according to the trauma group $+: p<0.05,++: p<0.01$, +++ : $p<0.001$, among treatment groups: \#: $p<0.05$, \# \#: $p<0.01$, \# \#: $p<0.001)$.
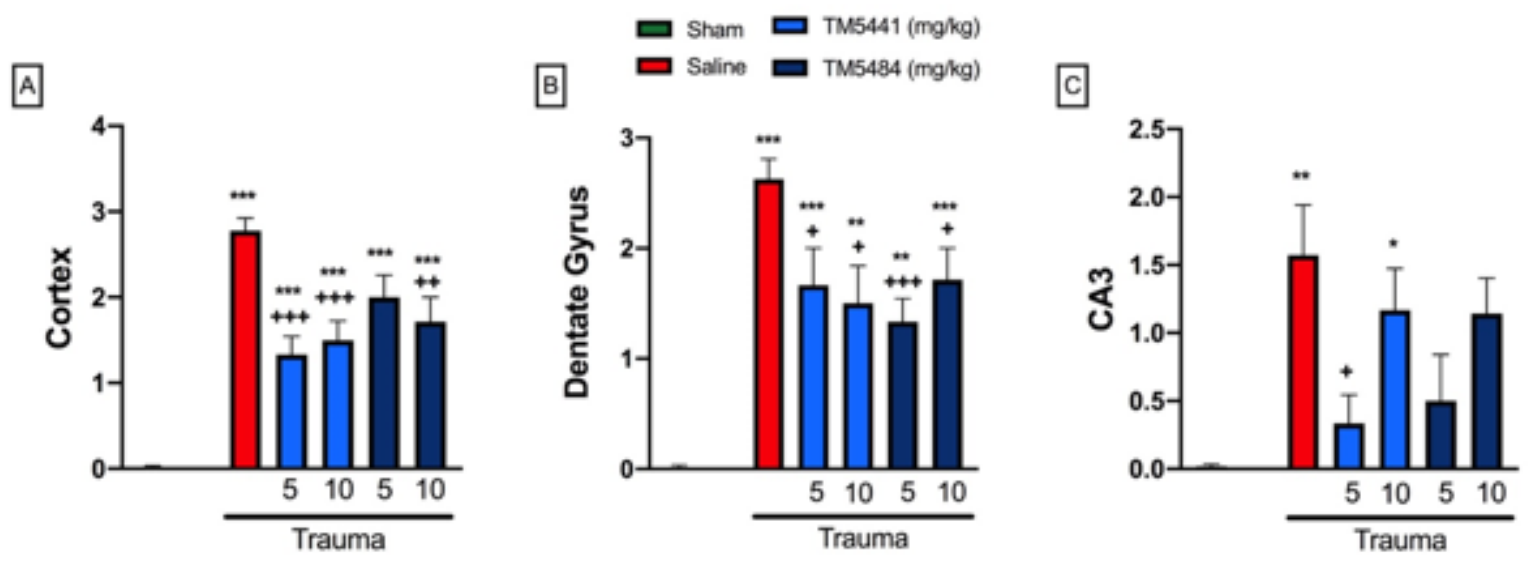

Figure 6

A) Cortex, B) Hippocampal dentate gyrus, C) Hippocampus CA3 region histological damage score among experimental groups (According to the sham group; $*$ : $<0.05, * *$ : $p<0.01, * \star *$ : $p<0.001$; according to the trauma group; + : $p<0.05,++$ : $p<0.01,+++$ : $p<0.001)$. 


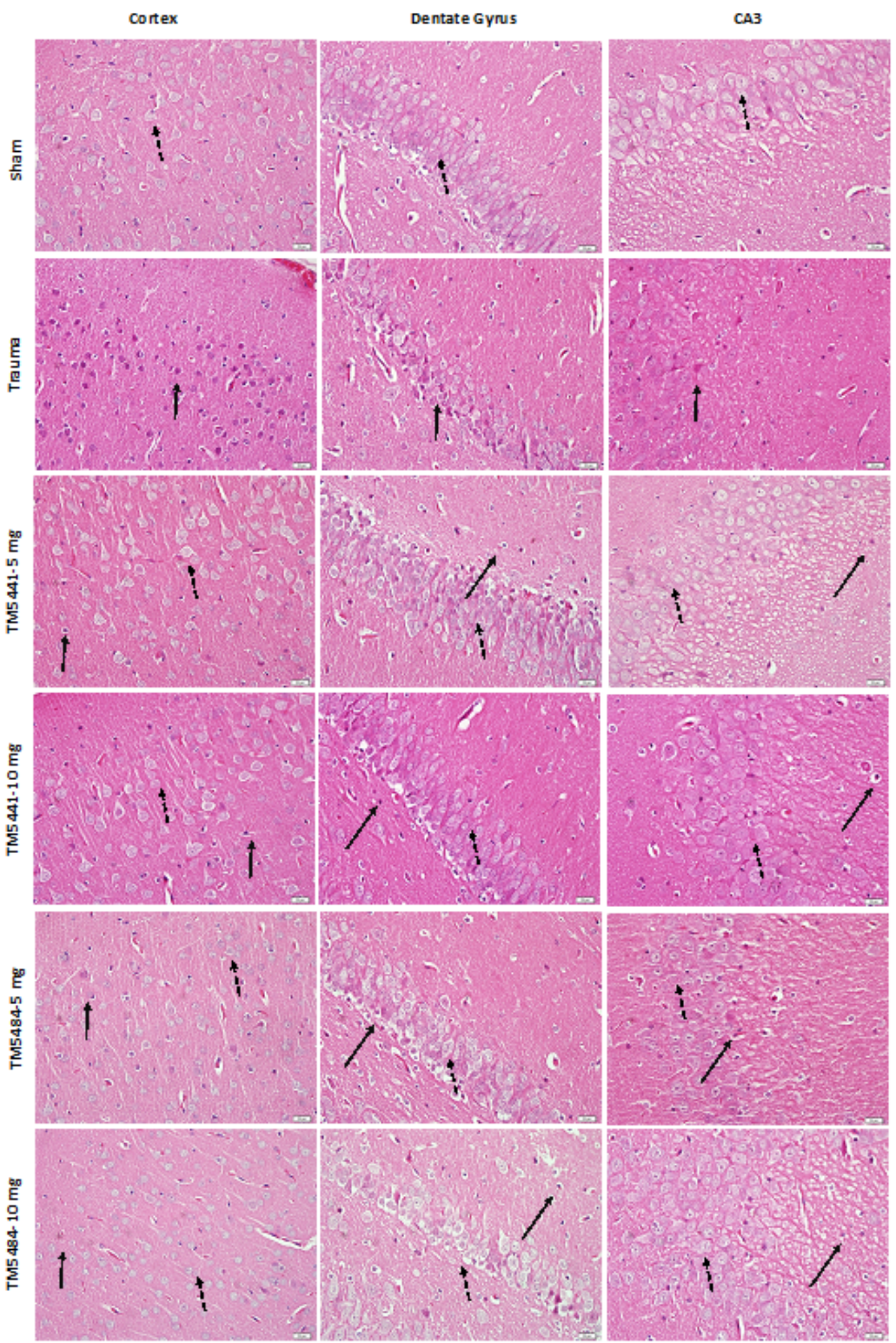

Figure 7

The histological examinations were performed with hematoxylin and eosin staining. Representative sections of the cortex, hippocampal dentate gyrus, and hippocampal CA3 region. The dashed black arrow indicates normal neuron morphology, the continuous black arrow indicates the damaged neuron containing a pycnotic cell nucleus (H\&E, 40X). 


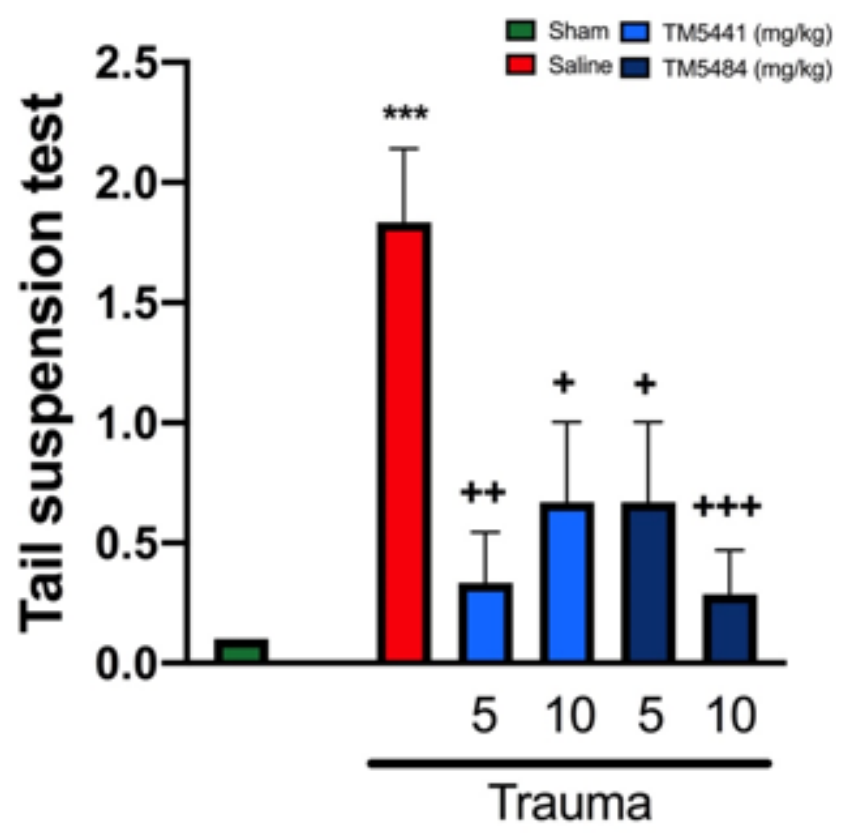

Figure 8

Tail suspension test scores among experimental groups (According to sham group ${ }^{\star \star \star}$ : $p<0.001$; according to trauma group $+: p<0.05,++: p<0.01,+++$ : $p<0.001)$.

\section{Supplementary Files}

This is a list of supplementary files associated with this preprint. Click to download.

- SupplementaryFigures.docx 